\title{
Using high pressure X-ray powder diffraction to exploring plastic and elastic deformations in ultra-hard transition metal borides
}

\author{
Sarah H. Tolberta,b,c \\ aDepartments of Chemistry and Biochemistry, UCLA, Los Angeles, CA 90095 \\ ${ }^{b}$ Department of Materials Science and Engineering, UCLA, Los Angeles, CA 90095 \\ 'The California NanoSystems Institute, UCLA, Los Angeles, CA 90095 \\ tolbert@chem.ucla.edu
}

In this talk, we will examine methods to measure and understand both plastic and elastic deformations in an every growing family of ultra-hard materials based on transition metal borides. We will specifically focus on the use of non-hydrostatic high pressure $\mathrm{X}$-ray diffraction methods to directly probe both elastic lattice deformations and the onset of plastic deformation in a wide range of materials in a lattice specific manner. Ultra-hard transition metal borides are exciting because, unlike diamond, they can be synthesized at atmospheric pressure. The materials were initially constructed using three very simple design rules: 1) use late transition metals to create high electron density so that the materials are incompressible; 2 ) add boron to build strong covalent bond to prevent slip and generate hard materials; 3 ) use solid-solution effects to further tune materials properties. In this talk, we exploit these simple ideas using a combination of materials synthesis, indentation measurements, and high pressure X-ray diffraction to gain an understanding of how the hardness in this family of materials can be tuned based on chemical composition and bonding motifs. To gain a global understanding of the family of materials we will move from metal $(M)$ rich $M B$ phases, to more conventional $\mathrm{MB}_{2}$ and $\mathrm{MB}_{4}$ type materials, and finally to very high boron content $\mathrm{MB}_{12}$ type materials. Finally, we will end with some new studies on nanoscale versions of these materials, where nanoscale architecture is combined with bonding constraints to further improve hardness. These nanoscale materials also allow for more detailed diffraction analysis and thus increased insight from the diffraction data. 